\title{
Charge ordering and magnetism in quarter-filled Hubbard-Holstein model
}

\author{
Sanjeev Kumar ${ }^{1,2}$ and Jeroen van den Brink ${ }^{1,3}$ \\ ${ }^{1}$ Institute Lorentz, for Theoretical Physics, Leiden University, P.O. Box 9506, 2300 RA Leiden, The Netherlands \\ ${ }^{2}$ Faculty of Science and Technology, University of Twente, P.O. Box 217, 7500 AE Enschede, The Netherlands \\ ${ }^{3}$ Institute for Molecules and Materials, Radboud Universiteit Nijmegen, P.O. Box 9010, 6500 GL Nijmegen, The Netherlands
}

(Received 23 May 2008; revised manuscript received 14 August 2008; published 23 October 2008)

\begin{abstract}
We study a two-dimensional Hubbard-Holstein model with phonons treated in the adiabatic limit. A HartreeFock decomposition is employed for the Hubbard term. A range of electronic densities are discussed with special emphasis on the quarter filling $(n=0.5)$. We argue that the quarter-filled system is relevant for the electronic properties observed at the interface between $\mathrm{LaAlO}_{3}$ and $\mathrm{SrTiO}_{3}$, where half-electron per unit cell is transferred to the $\mathrm{TiO}_{2}$ layer as a consequence of the polar discontinuity at the interface. In addition to presenting the overall phase diagrams, we identify an interesting charge-ordered antiferromagnetic phase for $n=0.5$, which was also reported recently in the $a b$ initio study of the $\mathrm{LaAlO}_{3}-\mathrm{SrTiO}_{3}$ interface.
\end{abstract}

DOI: 10.1103/PhysRevB.78.155123

PACS number(s): 71.10.Fd, 71.38.Ht

\section{INTRODUCTION}

The interfaces between bulk insulating oxides such as $\mathrm{LaAlO}_{3}$ (LAO) and $\mathrm{SrTiO}_{3}$ (STO) have recently become a topic of very active research. ${ }^{1-5}$ The interest in such interfaces was triggered by the observation of unusually high inplane conductivity at the $\mathrm{TiO}_{2}$-terminated interface. ${ }^{2}$ Materials with such high mobilities can find numerous applications in various fields of industry and can also be utilized to make new devices. ${ }^{3,4}$ The origin of this effect is believed to be an electronic reconstruction caused by the polar discontinuity at the interface. ${ }^{6}$ The conceptual idea is that in order to avoid a divergence of electrostatic potential, 0.5 electrons/unit cell are transferred to the $\mathrm{TiO}_{2}$ layer. These electrons then behave as a quasi-two-dimensional (2D) electron gas leading to large mobilities.

Subsequently, experiments were carried out at variable oxygen pressure and a strong dependence in the transport measurements was reported. ${ }^{7}$ In the presence of high-oxygen pressure, an insulating behavior in the resistivity was observed at low temperatures. This indicates that perhaps oxygen vacancies play a crucial role in the existence of highinterfacial conductivity and the system may actually be insulating in the absence of oxygen vacancies. In addition, an external magnetic field has a strong effect on transport. ${ }^{7}$ Applying magnetic field leads to a gain in the conductivity at low temperatures. An explanation for the insulating behavior of the resistivity was suggested to be connected to the magnetism and perhaps to the Kondo effect.

While the concept of electronic charge reconstruction or oxygen vacancies can provide a reasonable explanation for the observed conductivity, there is no rigorous understanding for the origin of magnetism at the interface. Hence, the understanding of magnetotransport is also rather incomplete. The present theoretical understanding of the interface properties has been largely based on the density-functional theory (DFT) calculations. Existence of a charge-ordered state has been predicted by these calculations. ${ }^{8}$ At the level of a minimal model, a charge-ordered state can be obtained within the extended Hubbard model. ${ }^{9}$ But, such a state should be nonmagnetic, suggesting that there might be an alternate mecha- nism active in these systems which leads to the charge ordering. Recent $\mathrm{LDA}+U$ (LDA is local-density approximation) studies of the LAO/STO interface have shown that structural distortions are present in the vicinity of the interface indicating the presence of an electron-lattice coupling. In this study a charge-ordered antiferromagnetic (AF) state was found to be the ground state. ${ }^{10}$

In this paper we study the two-dimensional HubbardHolstein model as a simplest model capturing the effects of both electron-electron and electron-lattice interactions. We find that the magnetic moments are formed as a consequence of polaron formation. These magnetic moments are found to be antiferromagnetically correlated at finite densities. At quarter filling, the ground state is antiferromagnetic and charge ordered in agreement with the findings of recent $\mathrm{LDA}+U$ calculations.

\section{MODEL AND METHOD}

We consider a one-band Hubbard-Holstein model on a square lattice with the Hamiltonian

$$
\begin{aligned}
H= & -t \sum_{\langle i j\rangle \sigma}\left(c_{i \sigma}^{\dagger} c_{j \sigma}+\text { H.c. }\right)+U \sum_{i} n_{i \uparrow} n_{i \downarrow} \\
& -\lambda \sum_{i} x_{i}\left(n_{i}-n\right)+\frac{K}{2} \sum_{i} x_{i}^{2} .
\end{aligned}
$$

Here, $c_{i \sigma}$ and $c_{i \sigma}^{\dagger}$ are the electron annihilation and creation operators with electronic spin $\sigma=\uparrow, \downarrow . n_{i}=n_{i \uparrow}+n_{i \downarrow}$ is the charge-density operator at site $i$ with $n_{i \sigma}=c_{i \sigma}^{\dagger} c_{i \sigma}$. The average electronic charge density is denoted by $n$ and $x_{i}$ denotes the volume contraction and expansion of the oxygen octahedra, which couples to the variations in the charge density. The hopping parameter $t$ is set to one, therefore all energy scales are in units of $t . U$ is the strength of on-site Hubbard repulsion and $\lambda$ is that of electron-lattice coupling. The lattice stiffness constant $K$ is set to one.

In the present study the lattice distortions $x_{i}$ are treated in the adiabatic limit. In the absence of the Hubbard term, the electronic Hamiltonian is bilinear in annihilation and creation operators, with the background potential provided by 
the configuration $\left\{x_{i}\right\}$ of lattice distortions. The ground state therefore corresponds to the lattice configuration which minimizes the total energy. In the absence of the kinetic-energy term $(t=0)$, the problem reduces to $N$ replicas of the one on a single site, where $N$ is the number of lattice sites. The total energy for a single site is given by

$$
E=-\lambda x_{i}\left(\left\langle n_{i}\right\rangle-n\right)+K x_{i}^{2} / 2 .
$$

Here and below $\langle A\rangle$ denotes the expectation value of the operator $A$. Minimization of the energy $E$ with respect to the classical variable $x_{i}$ leads to $x_{i}=(\lambda / K)\left(\left\langle n_{i}\right\rangle-n\right)$. For finite $t$, however, the kinetic-energy term also contributes to the total energy,

$$
E=-t \sum_{\langle i j\rangle \sigma}\left\langle c_{i \sigma}^{\dagger} c_{j \sigma}+\text { H.c. }\right\rangle-\lambda \sum_{i} x_{i}\left(\left\langle n_{i}\right\rangle-n\right)+\frac{K}{2} \sum_{i} x_{i}^{2} .
$$

Note that $x_{i}$ is not a specified potential but has to be determined self-consistently with the distribution of the electronic charge density. We compute the self-consistent lattice potential in the following scheme: Start with an arbitrary configuration of lattice distortions $\left\{x_{i}\right\}$. Diagonalize the Hamiltonian to generate the eigenvalues and eigenvectors. Compute the electronic charge density $\left\langle n_{i}\right\rangle$. Use the relation $x_{i}$ $=(\lambda / K)\left(\left\langle n_{i}\right\rangle-n\right)$ at each site to generate the new configuration for $\left\{x_{i}\right\}$. Repeat the process until the old and new charge densities match within given error bar.

In order to include the Hubbard term into the above selfconsistent formalism, we treat the Hubbard term within Hartree-Fock approximation. The Hartree-Fock decomposition of the Hubbard term leads to

$$
H_{U}=U \sum_{i}\left\langle n_{i \uparrow}\right\rangle n_{i \downarrow}+n_{i \uparrow}\left\langle n_{i \downarrow}\right\rangle-\left\langle n_{i \uparrow}\right\rangle\left\langle n_{i \downarrow}\right\rangle .
$$

Now the self-consistency cycle requires the convergence of $\left\langle n_{i \uparrow}\right\rangle$ and $\left\langle n_{i \downarrow}\right\rangle$ individually. The generic problem with the self-consistent methods is that they need not lead to the minimum-energy solution. Therefore, we use a variety of ordered and random initial states for the self-consistency loop and select the converged solution with the lowest energy.

The Hubbard-Holstein model contains a variety of interesting phases and phenomena including superconductivity, charge- and spin-density wave formations, phase separation, and polaron and bipolaron formations. For this reason, the Hubbard-Holstein model has always been of interest in different contexts. ${ }^{11-16}$ Most of the earlier studies on this model were focused at or near half filling. The quarter-filled case has not been analyzed in much detail except in one dimension. ${ }^{17}$

\section{RESULTS}

\section{A. Dilute limit}

We begin by analyzing the case with very few electrons. Consider Hamiltonian (1) with a single electron. The Hubbard term is inactive and for small $\lambda$ the ground-state wave
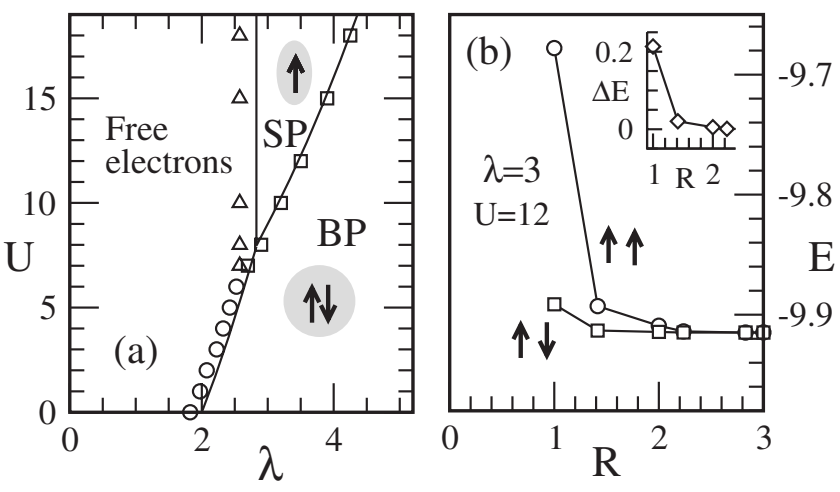

FIG. 1. (a) Phase diagram in the limit of low electron density in the parameter space of electron-lattice coupling $\lambda$ and the Hubbard repulsion $U$. Single polaronic and bipolaronic regimes are denoted by $\mathrm{SP}$ and $\mathrm{BP}$, respectively. The solid lines are from a strongtrapping analysis (see text) and the symbols are the results of numerical calculations. (b) Total energy as a function of the distance between two single polarons. The circles (squares) are for the parallel (antiparallel) spins of the single polarons. Inset shows the effective magnetic coupling between two self-trapped electrons as a function of the distance between them.

function corresponds to a Bloch wave. Since the lattice remains undistorted, i.e., $x_{i} \equiv 0$, the only contribution to the total energy is from the kinetic energy. For a single electron in a $2 \mathrm{D}$ square lattice, the lowest eigenvalue is $-4 t$, which is also equal to the total kinetic energy. Upon increasing the value of $\lambda$, the energy is gained via the Holstein coupling term by self-trapping of the electron into a single polaron (SP). In mean field the trapping occurs only when the energy of the SP state is lower than $-4 t$. Within a simple analysis, where we assume an ideal trapping of the electron at a single site, Eq. (2) leads to $E_{\mathrm{SP}}=-\lambda^{2} / K+\lambda^{2} /(2 K)=-\lambda^{2} /(2 K)$. Hence, the critical value of $\lambda$ required for trapping a single electron into a single polaron is given by $\lambda_{c}^{\mathrm{SP}}=\sqrt{8 K t}$.

Now consider the case of two electrons. In addition to the possibility of trapping the electrons as two single polarons, it is also possible to find a bipolaron (BP) solution. In fact the BP solution has a lower energy than two SPs and the critical value of $\lambda$ required to form a $\mathrm{BP}$ is given by $\lambda_{c}^{\mathrm{BP}}=\sqrt{4 K t}$. The tendency to form bipolarons is clearly suppressed by the repulsive energy cost of the Hubbard term. From a very simple analysis of the two electron case one obtains $E_{\text {free }} \sim-8 t$, $E_{\mathrm{BP}} \sim-2 \lambda^{2} / K+U$, and $E_{\mathrm{SP}} \sim-\lambda^{2} / K$. Looking for various energy crossings as a function of $\lambda$ and $U$ one obtains the phase diagram shown in Fig. 1(a) for the three phases considered above. The solid lines are from the simple analysis described above and the symbols represent the boundary values from the self-consistent numerical calculation. Note that this phase diagram corresponds to the case of two electrons in an infinite lattice and therefore refers to $n \rightarrow 0$ in terms of fractional electronic filling in the thermodynamic limit. The dilute limit of the Holstein model has been extensively studied in the context of polaron formation and self-trapping transitions. ${ }^{18-20}$ A variety of methods including weak- and strong-coupling perturbation theories, dynamical mean-field theory, and Monte Carlo simulations have been employed in the previous studies. Most of these studies were not re- 
stricted to the adiabatic limit; therefore a direct comparison of the present results is not possible. Nevertheless, we find that some of the features are very well reproduced by the present method, e.g., the self-trapping threshold $\lambda_{c}^{\mathrm{SP}} \sim 2.5$ compares very well with the values reported in previous studies. ${ }^{19,20}$

Assuming that $U$ is large so that we are in the regime of single polaron formation, we estimate the effective interaction between two single polarons by calculating the total energy as a function of the distance between them. The energy difference between the spin-aligned and spinantialigned single polarons provides an estimate for effective magnetic interaction between two polarons. The energy variations are shown in Fig. 1(b), suggesting a repulsive and antiferromagnetic interaction between the localized magnetic moments. The energy difference $\Delta E=E_{\uparrow \uparrow}-E_{\uparrow \downarrow}$ is plotted in the inset in Fig. 1(b). Positive values of $\Delta E$ for all $R$ show that the two trapped moments prefer to be antiferromagnetic for all distances. In fact, the strength of the interaction is almost vanishingly small for $R>2$, suggesting the absence of any ordered magnetic state for low densities. We will see in Secs. III B and III C that the above analysis of the dilute limit provides a very simple understanding of the phases that occur at finite densities, in addition to clarifying the basic competing tendencies present in the Hubbard-Holstein model.

\section{B. Generic electron densities}

For analyzing the system at higher electron densities we employ the self-consistent method described in Sec. III A. For the converged solution with minimum energy, we compute the charge structure factor,

$$
D_{n}(\mathbf{q})=N^{-2} \sum_{i j}\left(\left\langle n_{i}\right\rangle-n\right)\left(\left\langle n_{j}\right\rangle-n\right) e^{-\mathrm{i} \mathbf{q} \cdot\left(\mathbf{r}_{i}-\mathbf{r}_{j}\right)},
$$

and the spin structure factor,

$$
D_{s}(\mathbf{q})=N^{-2} \sum_{i j}\left\langle s_{i}\right\rangle\left\langle s_{j}\right\rangle e^{-\mathrm{i} \mathbf{q} \cdot\left(\mathbf{r}_{i}-\mathbf{r}_{j}\right)},
$$

with $s_{i}=\left(n_{i \uparrow}-n_{i \downarrow}\right) / 2$. Various ordered phases are inferred from the peaks in these structure factors. Figure 2(a) shows the spin structure factor at $\mathbf{q}=(\pi, \pi)$, which is a measure of antiferromagnetic correlations, and at $\mathbf{q}=(0,0)$, which is indicative of a ferromagnetic behavior. At $\lambda=0$ the system is antiferromagnetic (AFM) at and near $n=1$, it becomes ferromagnetic (FM) for $0.7<n<0.9$, and eventually becomes paramagnetic (PM). The antiferromagnetism at half filling arises as a consequence of the nesting feature of the Fermi surface. The ferromagnetism at intermediate densities can be understood within a Stoner picture which suggests that the repulsive cost coming from the Hubbard term can be reduced by a relative shift of the spin-up and spin-down bands. At $\lambda=2$, the antiferromagnetic regime near $n=1$ broadens [see Fig. 2(b)]. The ferromagnetism is absent. Near $n=0.5$ we find peaks in the charge structure factor at $(\pi, \pi)$, which indicates a charge-ordered $(\mathrm{CO})$ state. Simultaneous peaks are found in the spin structure factors at $(0, \pi)$ and $(\pi, 0)$ pointing toward the existence of a nontrivial state with si-
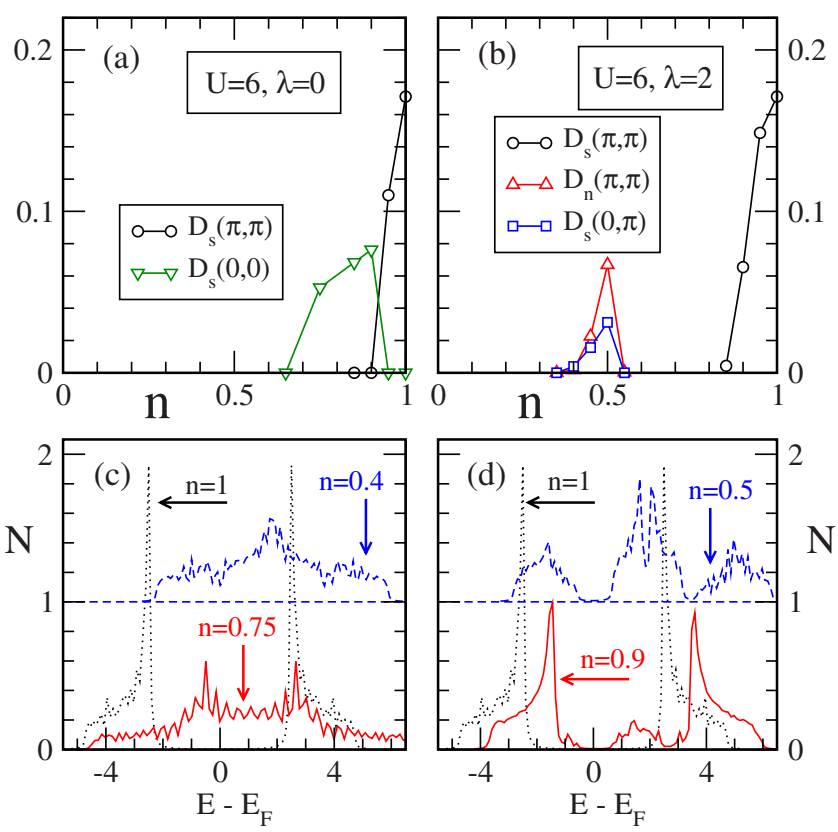

FIG. 2. (Color online) The charge and spin structure factors at various $\mathbf{q}$ as a function of average charge density $n$ at $U=6$ for (a) $\lambda=0$ and (b) $\lambda=2$. The total density of states for selected $n$ are shown for (c) $\lambda=0$ and (d) $\lambda=2$. The dotted (blue) curves in (c) and (d) are shifted along the $y$ axis for clarity.

multaneous existence of charge and spin ordering. All the results presented in this paper are for $N=32^{2}$; the stability of these results has been checked for system sizes up to $N$ $=40^{2}$.

To further analyze the nature of electronic states we compute the density of states (DOS) as

$$
N(\omega)=N^{-1} \sum_{i} \delta\left(\omega-\epsilon_{i}\right) \approx N^{-1} \sum_{i} \frac{\gamma / \pi}{\gamma^{2}+\left(\omega-\epsilon_{i}\right)^{2}} .
$$

Here, $\epsilon_{i}$ denote the eigenenergies corresponding to the minimum-energy configuration. The $\delta$ function is approximated by a Lorentzian with width $\gamma$. We use $\gamma=0.04$ in the calculations. A clean gap in the DOS is observed only for $n=1$ in the absence of $\lambda$ [see Fig. 2(c)]. In the FM regime, a two-peak structure represents a shifted spin-up and spindown band, which is consistent with the Stoner picture of magnetism in Hubbard model. Eventually at low density the DOS begins to resemble the free-electron tight-binding DOS. More interesting features are observed in the DOS at $\lambda=2$ shown in Fig. 2(d). The clean gap originating from the AFM state survives down to $n \sim 0.85$. The gap opens up once again at quarter filling $(n=0.5)$. This correlates perfectly with the signatures found in the structure factor calculations shown in Fig. 2(b).

The results for various $U$ at $\lambda=0$ and $\lambda=2$ are summarized into two phase diagrams. The $U-n$ phase diagram for $\lambda=0$ is shown in Fig. 3(a). Antiferromagnetic, ferromagnetic, and paramagnetic states are found to be stable in agreement with previous results on the Hubbard model in two dimensions. $^{21-23}$ Figure 3(b) shows the $U-n$ phase diagram for $\lambda=2$. For low $U$, the system becomes a bipolaronic insu- 

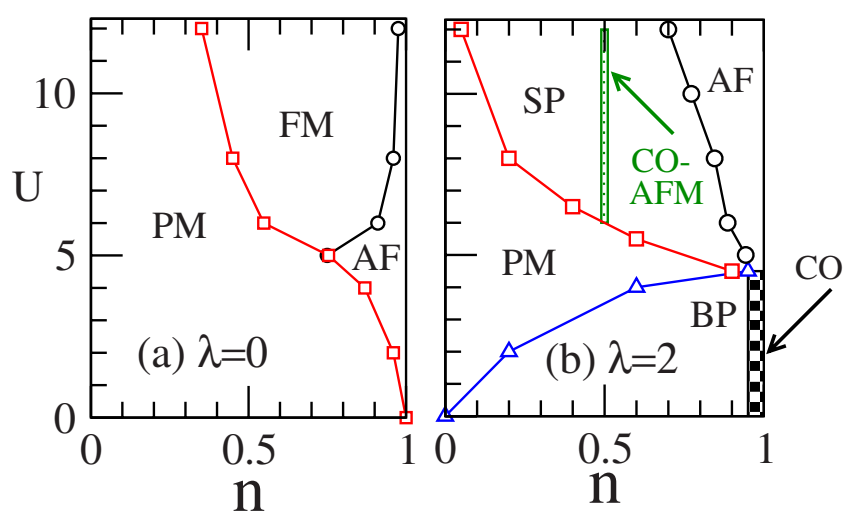

FIG. 3. (Color online) The $U-n$ phase diagrams for the HubbardHolstein model for (a) $\lambda=0$ and (b) $\lambda=2$. FM, AF, and PM phases are present in case of the pure Hubbard model $(\lambda=0)$. SP, BP, and charge-ordered antiferromagnetic phases also become stable for $\lambda$ $=2$.

lator. Although the $\lambda_{c}^{\mathrm{BP}} \sim 2$ for a single $\mathrm{BP}$, it can be much lower for finite density, suggesting that it is easier to trap many bipolarons as compared to a single BP. ${ }^{20}$ The chargeordered state at half filling can be simply viewed as a checkerboard arrangement of bipolarons, although the concept of an isolated BP does not really hold for such large densities. The charge-ordered state exists even below the critical coupling required for $\mathrm{BP}$ formation due to the nesting feature present in the Fermi surface at half filling. The half-filled $\mathrm{CO}$ state undergoes a transition to an antiferromagnetic state near $U=4$. The region of antiferromagnetism grows with increasing $U$ in contrast to the pure Hubbard model. The PM state still exists for small $\lambda$ but the FM state is absent. A large region of phase space is taken by the single polaronic state for large $U$. No magnetism is found at low densities, since these single polarons are magnetically noninteracting due to the large interpolaronic separations. At large densities, however, there are antiferromagnetic correlations between these single polarons. This is consistent with the effective magnetic interactions found between two single polarons [see Fig. 1(b)]. These effective antiferromagnetic interactions are the origin of the growth in the AFM regime near $n=1$.

\section{Half and quarter filling}

The Hubbard-Holstein model at and near half filling has been studied previously. ${ }^{24-26}$ The existence of spin- and charge-density waves was reported. The possibility for an intermediate metallic phase was also reported in a onedimensional model with dynamical effects for lattice. ${ }^{16}$ Figure 4(a) shows a $U$ - $\lambda$ phase diagram at half filling. The system is either charge ordered or antiferromagnetic and, therefore, the DOS is always gapped. The boundary separating the $\mathrm{CO}$ and the AFM states fits very well a $U=\lambda^{2}$ power law, which happens to be the boundary separating the SP and $\mathrm{BP}$ regimes in the low-density limit [see Fig. 1(a)]. This suggests that the $\mathrm{CO}$ phase can be viewed as a checkerboard pattern of bipolarons, at least for large values of $\lambda$. The origin of the CO or the AFM phase at small values of $U$ and $\lambda$
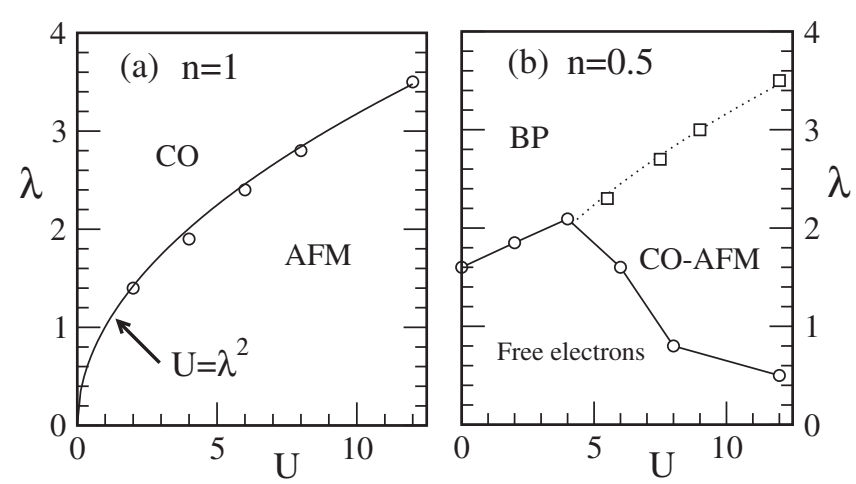

FIG. 4. $\lambda-U$ phase diagrams at (a) half filling and (b) quarter filling.

is related to the existence of nesting in the Fermi surface with a nesting wave vector $\mathbf{q}=(\pi, \pi)$.

The most interesting result of this paper is the observation of a CO-AFM state at quarter filling. We plot the $U-\lambda$ phase diagram for quarter-filled system in Fig. 4(b). Unlike the half-filled case, the small $U$ and small $\lambda$ regimes correspond to free-electron behavior. The SP state is found to exist for large $U$ and there is a large window where a charge-ordered AFM state exists. We find that a self-consistent solution corresponding to a CO-FM state can exist only for $U>10$, but it is still higher in energy than the CO-AFM. For $U<10$, the CO-FM state is not stable and therefore the charge ordering occurs only when it is accompanied by an AFM ordering. This leads to a very interesting implication for the effects of external magnetic field. Destabilizing the AFM phase by applying an external magnetic field to the CO-AFM state would lead to a melting of the charge order and, hence, a collapse of the gap in the density of states. The limiting cases of half and quarter filling have been studied before in one dimension using quantum Monte Carlo method. ${ }^{17}$ The phase diagram at half filling contains an intermediate metallic

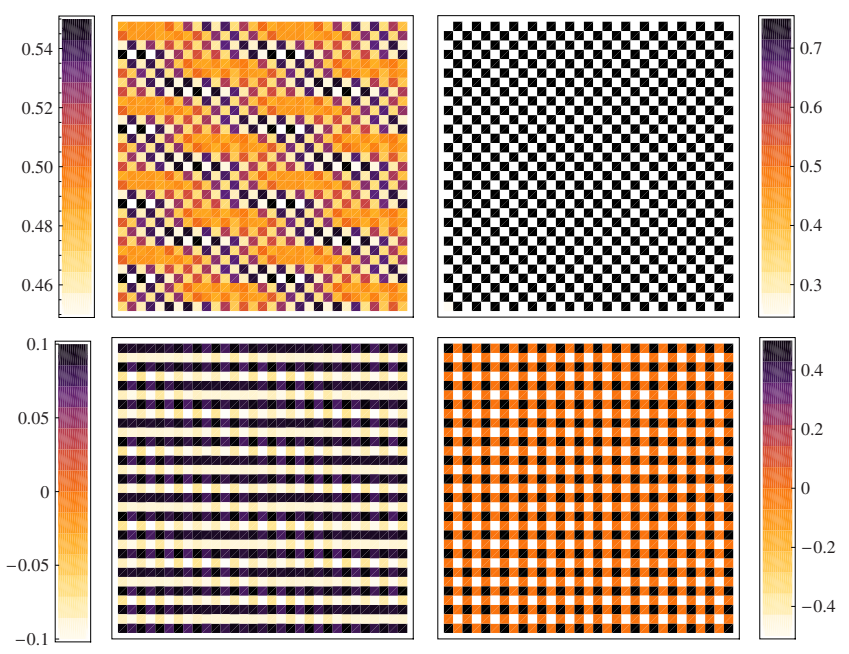

FIG. 5. (Color online) Real-space patterns for charge density (upper row) and spin density (lower row) at $U=6$ and $\lambda=1$ (left column) and $\lambda=2$ (right column). Note that the spin state for $\lambda=2$ is a $G$-type antiferromagnet if one rotates the lattice by $45^{\circ}$ and consider the square lattice of occupied sites. 
phase, in addition to the two phases discussed above. However, the region in parameter space of this intermediate metallic phase shrinks as one approaches the adiabatic limit, leading to a phase diagram very similar to ours [see Fig. 4(a)]. Therefore, the dimensionality does not appear to be very crucial for understanding the half-filled case. The quarter-filled case in Ref. 17 is found to have essentially the same phases as in the half-filled case. The coexisting COAFM state that we report in the present study was not observed, suggesting that the higher dimension may be crucial for the stability of this state.

To further investigate this unusual state at quarter filling, we show the real-space data for charge density $n_{i}$ and spin density $s_{i}$ in Fig. 5. A weak charge ordering is already present at $\lambda=1$ (see upper-left panel). This charge ordering is accompanied by a stripelike spin ordering, where spins are arranged ferromagnetically along one direction and antiferromagnetically along the other (lower-left panel). A very clear CO pattern emerges for the larger value of $\lambda$ (upperright panel), which occurs together with an AFM arrangement of the spins (lower-right panel). In a strong-coupling scenario, it is easy to understand the CO-AFM state within the picture of effective magnetic interaction between single polarons presented in Fig. 1(b). Assuming that the "occupied" sites in the charge-ordered state can be viewed as single polarons, an effective antiferromagnetic interaction between them is strongest at distance $\sqrt{2}$, hence leading to a magnetic structure which is a $G$-type AFM order for the square lattice constructed out of the occupied sites only. For smaller values of $\lambda$ the charge disproportionation in the $\mathrm{CO}$ state is much smaller and the effective magnetic interaction picture cannot be pushed to this weak-coupling regime.

\section{CONCLUSIONS}

We have presented ground-state properties of the Hubbard-Holstein model in two dimensions in the adiabatic limit for the lattice distortions. We use a self-consistent method for generating the static lattice configurations in combination with a Hartree-Fock decoupling of the Hubbard term. Interestingly, the charge-ordered antiferromagnetic state that we find at quarter filling was shown to be the ground state for the LAO/STO interface in recent DFT calculations. ${ }^{10}$ Within our analysis the charge ordering in this state occurs only in combination with the AFM ordering, as we find that the CO-FM state is unstable. Therefore, the charge ordering could be melted by applying an external magnetic field leading to a large negative magnetoresistance. We argue that this model is relevant for the LAO/STO interface since (i) it provides a possibility for the formation of magnetic moments, (ii) leads to a CO-AFM ground state in agreement with the recent $\mathrm{LDA}+U$ studies, and (iii) contains the possibility for large negative magnetoresistance via a magnetic-field-induced melting of the charge-ordered state.

\section{ACKNOWLEDGMENTS}

We gratefully acknowledge useful discussions with Z. Zhong, G. Brocks, and P. J. Kelly. This work was financially supported by "NanoNed," a nanotechnology program of the Dutch Ministry of Economic Affairs, and by the "Nederlandse Organisatie voor Wetenschappelijk Onderzoek (NWO)" and the "Stichting voor Fundamenteel Onderzoek der Materie (FOM)."
${ }^{1}$ A. Ohtomo, D. A. Muller, J. L. Grazul, and H. Y. Hwang, Nature (London) 419, 378 (2002).

${ }^{2}$ A. Ohtomo and H. Y. Hwang, Nature (London) 427, 423 (2004).

${ }^{3}$ S. Thiel, G. Hammerl, A. Schmehl, C. W. Schneider, and J. Mannhart, Science 313, 1942 (2006).

${ }^{4}$ C. W. Schneider, S. Thiel, G. Hammerl, C. Richter, and J. Mannhart, Appl. Phys. Lett. 89, 122101 (2006).

${ }^{5}$ N. Reyren, S. Thiel, A. D. Caviglia, L. Fitting Kourkoutis, G. Hammer, C. Richter, C. W. Schneider, T. Kopp, A.-S. Retschi, D. Jaccard, M. Gabay, D. A. Muller, J.-M. Triscone, and J. Mannhart, Science 317, 1196 (2007).

${ }^{6}$ N. Nakagawa, H. Y. Hwang, and D. A. Muller, Nature Mater. 5, 204 (2006).

${ }^{7}$ A. Brinkman, M. Huijben, M. van Zalk, J. Huijben, U. Zeitler, J. C. Maan, W. G. van der Wiel, G. Rijnders, D. H. A. Blank, and H. Hilgenkamp, Nature Mater. 6, 493 (2007).

${ }^{8}$ R. Pentcheva and W. E. Pickett, Phys. Rev. B 74, 035112 (2006).

${ }^{9}$ N. Pavlenko and T. Kopp, Phys. Rev. Lett. 97, 187001 (2006).

${ }^{10}$ Z. Zhong and P. J. Kelly, EPL 84, 27001 (2008).

${ }^{11}$ C.-H. Pao and H.-B. Schüttler, Phys. Rev. B 57, 5051 (1998).

${ }^{12}$ A. Macridin, G. A. Sawatzky, and M. Jarrell, Phys. Rev. B 69, 245111 (2004).
${ }^{13}$ M. Capone, P. Carta, and S. Ciuchi, Phys. Rev. B 74, 045106 (2006).

${ }^{14}$ M. Capone, G. Sangiovanni, C. Castellani, C. Di Castro, and M. Grilli, Phys. Rev. Lett. 92, 106401 (2004).

${ }^{15}$ Y. Takada and A. Chatterjee, Phys. Rev. B 67, 081102(R) (2003).

${ }^{16}$ R. T. Clay and R. P. Hardikar, Phys. Rev. Lett. 95, 096401 (2005).

${ }^{17}$ R. P. Hardikar and R. T. Clay, Phys. Rev. B 75, 245103 (2007).

${ }^{18}$ S. Ciuchi, F. de Pasquale, S. Fratini, and D. Feinberg, Phys. Rev. B 56, 4494 (1997).

${ }^{19}$ A. H. Romero, D. W. Brown, and K. Lindenberg, Phys. Rev. B 60, 14080 (1999).

${ }^{20}$ B. P. Sekhar, S. Kumar, and P. Majumdar, Europhys. Lett. 68, 564 (2004).

${ }^{21}$ J. E. Hirsch, Phys. Rev. B 31, 4403 (1985).

${ }^{22}$ W. Nolting and W. Borgiel, Phys. Rev. B 39, 6962 (1989).

${ }^{23}$ S. R. White, D. J. Scalapino, R. L. Sugar, E. Y. Loh, J. E. Gubernatis, and R. T. Scalettar, Phys. Rev. B 40, 506 (1989).

${ }^{24}$ A. Muramatsu and W. Hanke, Phys. Rev. B 38, 878 (1988).

${ }^{25}$ J. Zhong and H.-B. Schüttler, Phys. Rev. Lett. 69, 1600 (1992).

${ }^{26}$ E. Berger, P. Valásek, and W. von der Linden, Phys. Rev. B 52, 4806 (1995). 